\title{
DETERMINACIÓN DEL ÍNDICE DE COMPRESIÓN A TRAVÉS DE LAS PROPIEDADES DE PLASTICIDAD DE LOS SUELOS DE LA CIUDAD DE OCAÑA, NORTE DE SANTANDER
}

\section{DETERMINATION OF THE COMPRESSION INDEX THROUGH THE PLASTICITY PROPERTIES OF THE SOILS OF THE CITY OF OCAÑA, NORTE DE SANTANDER}

\author{
Ing. Luis Miguel Duarte Vergara*, Ing. Hugo Andrés Álvarez Quintero** \\ MSc. Romel Jesús Gallardo Amaya ${ }^{* * *}$ \\ * Universidad Francisco de Paula Santander Ocaña, Grupo de Investigación en \\ Construcción, Geotecnia y Medio Ambiente GIGMA. Vía Acolsure, Sede el Algodonal, \\ Ocaña, Norte de Santander, Colombia, \\ Email:1mduartev@ufpso.edu.co, haalvarezq@ufpso.edu.co,rjgallardoa@ufpso.edu.co
}

\begin{abstract}
Resumen: Uno de los parámetros más importantes para la determinación de los asentamientos por consolidación es el índice de compresibilidad, su determinación en laboratorio conlleva un tiempo considerable y por ello es común su cálculo usando ecuaciones que involucran las propiedades índice del suelo. La investigación realizada muestra los resultados obtenidos al correlacionar el índice de compresión con las propiedades de plasticidad de los suelos residuales de la ciudad de Ocaña, Norte de Santander, derivados de una formación geológica de origen ígneo (Complejo Intrusivo Extrusivo), presentes en las zonas de mayor expansión urbana. Se obtuvieron correlaciones para el índice de compresión con los límites de plasticidad de los suelos, siendo la correlación con el límite plástico la que mejor coeficiente de Pearson presentó, para el límite líquido los resultados son altamente confiables, mientras que el índice de plasticidad es la propiedad menos confiable con un bajo coeficiente de Pearson.
\end{abstract}

Palabras clave: Consolidación, correlación, índice de compresión, plasticidad.

Abstract: One of the most important parameters for the determination of settlements is the compressibility index, its determination in the laboratory takes considerable time and therefore its calculation is common using equations that involve the soil index properties. The research carried out shows the results obtained by relating the compression rate with the plasticity properties of the residual soils of the city of Ocaña, Norte de Santander, derived from a geological formation of igneous origin (Intrusive - Extrusive Complex), present in the areas of greatest urban expansion. Correlations were obtained for the compression index with the plasticity limits of the soils, the correlation with the plastic limit being the one that Pearson's best coefficient presented, for the liquid limit the results are highly reliable, while the plasticity index is the less reliable property with a low Pearson coefficient.

Keywords: Consolidation, correlation, compression rate, plasticity. 


\section{INTRODUCCIÓN}

La teoría de la consolidación propuesta por Karl Terzaghi, permitió describir el proceso de consolidación unidimensional y los parámetros que pueden obtenerse de éste (Juárez y Rico, 2012; Xiao, 2015). Dentro de estos parámetros se encuentran el coeficiente de compresibilidad $\left(a_{v}\right)$, el coeficiente de compresibilidad volumétrica $\left(\mathrm{m}_{\mathrm{v}}\right)$, el coeficiente de consolidación $\left(\mathrm{C}_{\mathrm{v}}\right)$, el coeficiente de permeabilidad $(\mathrm{k})$, el índice de compresión $\left(\mathrm{C}_{\mathrm{c}}\right)$ y el índice de expansión $\left(\mathrm{C}_{\mathrm{s}}\right)$. La aplicación de esta teoría permitió ir reemplazando paulatinamente los métodos empíricos que hasta entonces se utilizaban para la obtención de asentamientos en el suelo (Crespo, 2004; Das, 2015); (L Tangarife et al., 2017).

Dado que todos los suelos sufren algún tipo de deformación bajo el efecto de un esfuerzo aplicado, los parámetros de consolidación permiten determinar la magnitud de estas deformaciones y el comportamiento que presentan ante estos esfuerzos (Cuanalo et al., 2016; Molina et al., 2012); (J Plaza, M Núñez, 2017).

Los parámetros de consolidación pueden obtenerse a partir del ensayo de consolidación unidimensional en el laboratorio, sin embargo, esto demanda una inversión considerable de tiempo, llegando a tardar un ensayo entre 1 y 3 semanas (Ayala, 2013). Ante esta demanda de tiempo, diversos investigadores correlacionaron algunos parámetros de consolidación, especialmente el índice de compresión, con las propiedades de plasticidad del suelo, como el límite líquido, el límite plástico y el índice de plasticidad (Acevedo, et al., 2014; Gallardo et al., 2018); (R García León, et al., 2016).

La importancia de estos parámetros se ve reflejado en las diversas investigaciones realizadas para facilitar su obtención, es así que investigadores como Terzaghi y Peck (1967), Nishida (1956), Azzouz (1976), Koppula (1981), entre otros, plantearon correlaciones para suelos con determinadas características y en zonas delimitadas.

El índice de compresión es uno de los parámetros de consolidación más estudiados en la actualidad, pues es empleado en las ecuaciones establecidas para la obtención de asentamientos.
El objetivo principal de esta investigación fue determinar el índice de compresión a través de las propiedades de plasticidad de los suelos de la ciudad de Ocaña, Norte de Santander, para brindar una herramienta de apoyo en la realización de estudios geotécnicos.

\section{METODOLOGÍA}

Se trata de una investigación de tipo cuantitativo y experimental, que tomó como población de estudio los suelos derivados de una formación geológica de tipo ígneo conocida como el Complejo Intrusivo Extrusivo y, que está presente en un área considerable del municipio de Ocaña, Norte de Santander.

La figura 1 muestra las zonas de la ciudad de Ocaña (color rojo) donde se tiene afloramiento de la formación ígnea.

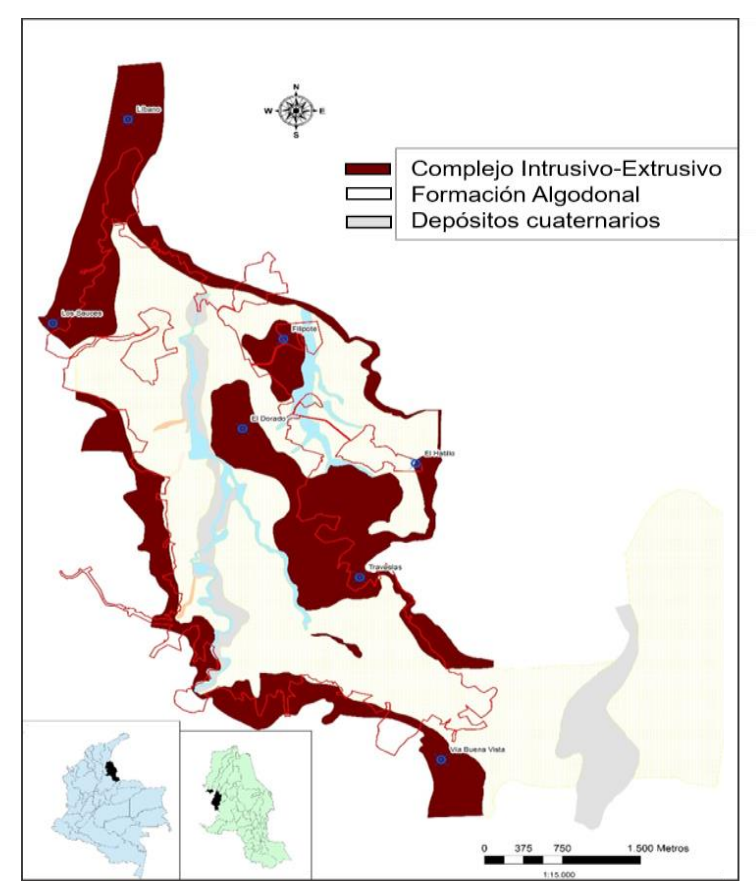

Fig. 1. Mapa geológico de la ciudad de Ocaña, Norte de Santander. Fuente: Adaptado de PBOT Ocaña 2015.

Teniendo en cuenta el mapa de la figura 1 se realizaron visitas a diferentes sectores de la ciudad, en zonas de expansión urbana, para seleccionar los sitios para la toma de muestras, verificando que se encontrarán perfiles de suelos residuales derivados de la formación geológica de interés. En la Tabla 1 se muestran las coordenadas de los puntos donde 
se realizó la toma de muestras alteradas e inalteradas de suelo empleando tubos Shelby.

Tabla 1: Localización de los puntos de muestreo

\begin{tabular}{cccc}
\hline \multirow{2}{*}{ Sector o Barrio } & \multicolumn{2}{c}{ Coordenadas } & Altitud \\
& Latitud & Longitud & (msnm) \\
\hline Los Sauces & 8.263 .943 & 73.372 .583 & 1.190 \\
Vía Buena Vista & 8.220 .824 & 73.338 .770 & 1.278 \\
Travesías & 8.238 .828 & 73.345 .855 & 1.267 \\
Filipote & 8.262 .370 & 73.352 .471 & 1.165 \\
El Dorado & 8.253 .528 & 73.356 .048 & 1.191 \\
El Hatillo & 8.250 .069 & 73.340 .949 & 1.208 \\
Líbano & 8.284 .063 & 73.365 .982 & 1.140 \\
\hline
\end{tabular}

Fuente: Autores.

\subsection{Formación geológica}

El Complejo Intrusivo - Extrusivo, hace parte del complejo ígneo metamórfico denominado "Macizo Santander" e incluye rocas ígneas de juratriásico (entre 144 y 245 MA.). Está constituido por una fase intrusiva cuya composición predominante es cuarzo - monzonita, pórfidos riolíticos, diques básicos de diabasas y basalto; también incluye material piroclástico con presencia de toba, brechas y aglomerados (SGC, 2007). Este tipo de formación se caracteriza por tener perfiles de meteorización con presencia de suelos residuales (Gallardo et al., 2017; García et al., 2018). En la figura 2 puede observase un perfil típico de esta formación.

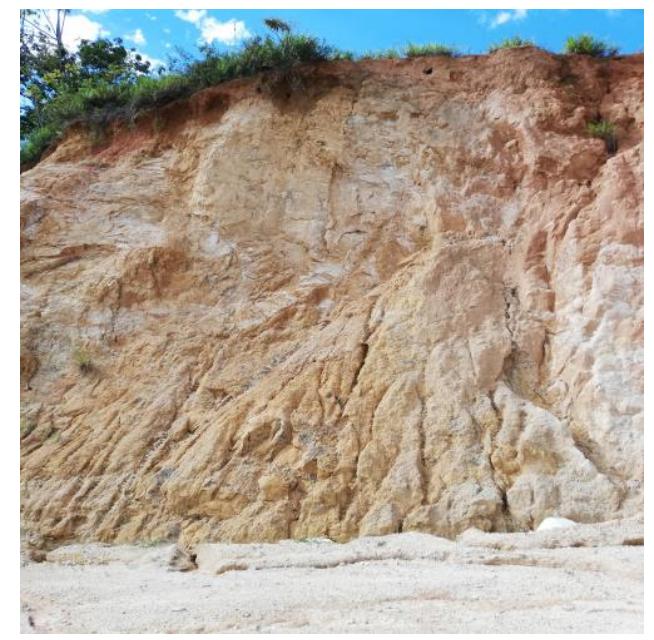

Fig. 2. Perfil de meteorización típico de los suelos de la formación ígnea presente en la ciudad de Ocaña. Fuente: Autores.
En algunos sectores de la ciudad esta formación genera suelos residuales de tipo arenoso de poco espesor debido a que sufren fuertes procesos de erosión. En otros sectores como los de expansión urbana los perfiles de meteorización son de mayor espesor y contienen suelos de tipo cohesivo (Sowers y Sowers, 1972).

\subsection{Características físicas del suelo}

Como una etapa importante de la investigación se requirió conocer las características de composición granulométrica y de plasticidad de los suelos obtenidos en los diferentes puntos de muestreo, esto con el fin de tener una clasificación basada en sistemas reconocidos comúnmente (Peck et al., 2004).

Se realizaron ensayos de clasificación y límites de consistencia de acuerdo a las indicaciones establecidas por el Instituto Nacional de Vías en la sección 100 de las Normas de Ensayo para Materiales (INVIAS, 2012). Los resultados de estos ensayos para cada muestra (Sector o barrio) se muestran en la tabla 2.

\section{Tabla 2: Resumen de los resultados de clasificación del suelo}

\begin{tabular}{|c|c|c|c|c|c|c|}
\hline & \multirow{2}{*}{$\begin{array}{c}\% \\
\text { Humedad }\end{array}$} & \multicolumn{3}{|c|}{$\begin{array}{l}\text { Propiedades de } \\
\text { plasticidad }\end{array}$} & \multirow{2}{*}{$\begin{array}{c}\% \\
\text { Pasa } \\
200\end{array}$} & \multirow{2}{*}{$\begin{array}{c}\text { Clasificación } \\
\text { S.U.C.S. }\end{array}$} \\
\hline & & $\% \mathrm{LL}$ & $\% \mathrm{LP}$ & $\%$ IP & & \\
\hline $\begin{array}{l}\text { Los Sauces } \\
\text { Vía Buena }\end{array}$ & 28,18 & 47,42 & 37,55 & 9,87 & 64,05 & ML \\
\hline Vista & 10,59 & 52,47 & 32,87 & 19,6 & 92,59 & MH \\
\hline Travesías & 23,37 & 48,7 & 36,58 & 12,12 & 61,94 & ML \\
\hline Filipote & 12,88 & 35,1 & 24,54 & 10,56 & 51,55 & ML \\
\hline El Dorado & 25,06 & 40,82 & 29,79 & 11,03 & 66,02 & ML \\
\hline El Hatillo & 16,63 & 37,82 & 27,8 & 10,02 & 71,63 & ML \\
\hline Líbano & 16,27 & 32,15 & 25,44 & 6,71 & 50,14 & ML \\
\hline
\end{tabular}

Fuente: Autores.

\subsection{Ensayos de consolidación unidimensional}

Con el ensayo de consolidación unidimensional se busca generar en laboratorio, las condiciones de un suelo de superficie horizontal cargado uniformemente, de tal forma que la única deformación posible sea en la dirección vertical (Sáez, 2010). Los resultados del ensayo permiten determinar el tiempo en el cual se produce la consolidación, además de la magnitud del asentamiento que tendrá lugar debido a la deformación del suelo (Beltrán, 2015). 
El objetivo de este ensayo fue determinar el índice de compresión de las muestras obtenidas en cada sector de muestreo, para ello se realizaron tres pruebas de consolidación unidimensional por cada sector, para un total de 21 ensayos, en la tabla 3 se muestran los valores del índice de compresión obtenidos.

Los ensayos de consolidación unidimensional y de clasificación del suelo fueron realizados en su totalidad en el laboratorio de suelos de la Universidad Francisco de Paula Santander Ocaña.

\section{Tabla 3: Valores de Cc obtenidos en los ensayos} de consolidación unidimensional

\begin{tabular}{|c|c|c|}
\hline Sector & $\begin{array}{l}\mathrm{N}^{\circ} \text { de } \\
\text { Ensayo }\end{array}$ & $\mathrm{Cc}$ \\
\hline \multirow{3}{*}{ Los Sauces } & 1 & 0,440 \\
\hline & 2 & 0,458 \\
\hline & 3 & 0,448 \\
\hline \multirow{3}{*}{ Vía Buena Vista } & 4 & 0,419 \\
\hline & 5 & 0,429 \\
\hline & 6 & 0,432 \\
\hline \multirow{3}{*}{ Travesías } & 7 & 0,441 \\
\hline & 8 & 0,459 \\
\hline & 9 & 0,464 \\
\hline \multirow{3}{*}{ Filipote } & 10 & 0,374 \\
\hline & 11 & 0,377 \\
\hline & 12 & 0,380 \\
\hline \multirow{3}{*}{ El Dorado } & 13 & 0,388 \\
\hline & 14 & 0,394 \\
\hline & 15 & 0,396 \\
\hline \multirow{3}{*}{ El Hatillo } & 16 & 0,371 \\
\hline & 17 & 0,348 \\
\hline & 18 & 0,348 \\
\hline \multirow{3}{*}{ Líbano } & 19 & 0,341 \\
\hline & 20 & 0,332 \\
\hline & 21 & 0,341 \\
\hline
\end{tabular}

Fuente: Autores..

\section{RESULTADOS}

En la tabla 4 se muestran los resultados de las propiedades de plasticidad y el índice de compresión obtenido en cada sector o barrio.

Con estos resultados se buscó establecer distintas correlaciones entre el índice de compresión y las propiedades de plasticidad, para de esta forma determinar cuál propiedad es la más adecuada para obtener el índice de compresión para los suelos cohesivos analizados.

Los gráficos y correlaciones fueron obtenidos mediante la herramienta ofimática Excel. Para todos los datos se aplicó el método de regresión lineal (Johnson, 2003).

\section{Tabla 4: Resumen de los resultados de laboratorio}

\begin{tabular}{|c|c|c|c|c|}
\hline \multirow{2}{*}{ Sector } & \multicolumn{3}{|c|}{ Propiedades de plasticidad } & \multirow{2}{*}{$\mathrm{Cc}$} \\
\hline & $\% \mathrm{LL}$ & $\%$ LP & $\%$ IP & \\
\hline \multirow{3}{*}{ Los Sauces } & \multirow{3}{*}{47,42} & \multirow{3}{*}{37,55} & \multirow{3}{*}{9,87} & 0,440 \\
\hline & & & & 0,458 \\
\hline & & & & 0,448 \\
\hline \multirow{3}{*}{ Vía Buena Vista } & \multirow{3}{*}{52,47} & \multirow{3}{*}{32,87} & \multirow{3}{*}{19,6} & 0,419 \\
\hline & & & & 0,429 \\
\hline & & & & 0,432 \\
\hline \multirow{3}{*}{ Travesías } & \multirow{3}{*}{48,7} & \multirow{3}{*}{36,58} & \multirow{3}{*}{12,12} & 0,441 \\
\hline & & & & 0,459 \\
\hline & & & & 0,464 \\
\hline \multirow{3}{*}{ Filipote } & \multirow{3}{*}{35,1} & \multirow{3}{*}{24,54} & \multirow{3}{*}{10,56} & 0,374 \\
\hline & & & & 0,377 \\
\hline & & & & 0,380 \\
\hline \multirow{3}{*}{ El Dorado } & \multirow{3}{*}{40,82} & \multirow{3}{*}{29,79} & \multirow{3}{*}{11,03} & 0,388 \\
\hline & & & & 0,394 \\
\hline & & & & 0,396 \\
\hline \multirow{3}{*}{ El Hatillo } & \multirow{3}{*}{37,82} & \multirow{3}{*}{27,8} & \multirow{3}{*}{10,02} & 0,371 \\
\hline & & & & 0,348 \\
\hline & & & & 0,348 \\
\hline \multirow{3}{*}{ Líbano } & \multirow{3}{*}{32,15} & \multirow{3}{*}{25,44} & \multirow{3}{*}{6,71} & 0,341 \\
\hline & & & & 0,332 \\
\hline & & & & 0,341 \\
\hline
\end{tabular}

\subsection{Correlación entre el índice de compresión y el límite líquido}

Con los datos de la tabla 4 se obtuvo el gráfico mostrado en la figura 3 .

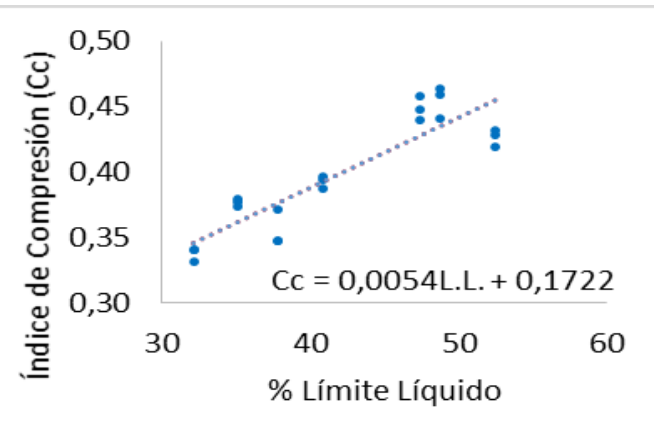


Fig. 3. Correlación entre índice de compresión y el límite líquido. Fuente: Autores.

Del gráfico anterior se obtiene la ecuación de correlación (1):

$$
\mathrm{Cc}=0,0054 *(\text { L.L. }+32)
$$

En el análisis realizado entre el índice de compresión y el límite líquido se obtuvo una expresión con un coeficiente de correlación lineal de Pearson $r=0,89$, que permite clasificar el grado de correlación como positiva alta (Johnson, 2003).

\subsection{Correlación entre el índice de compresión y el límite plástico}

Con los datos de la tabla 4 , se obtuvo el gráfico mostrado en la figura 4.

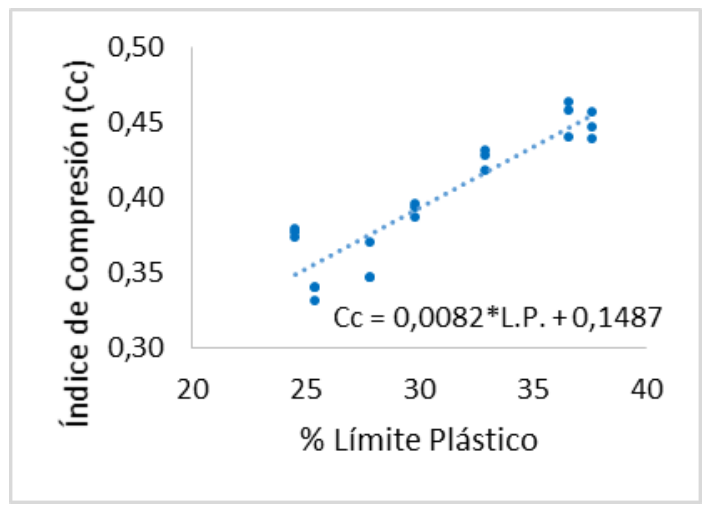

Fig. 4. Correlación entre índice de compresión y el límite plástico. Fuente: Autores.

Del gráfico anterior se obtiene la ecuación de correlación (2):

$$
\text { Cc }=0,0082 *(\text { L.P. }+18,13)
$$

En el análisis realizado entre el índice de compresión y el límite plástico se obtuvo una expresión con un coeficiente de correlación lineal de Pearson $r=0,92$, que permite clasificar el grado de correlación como positiva muy alta (Johnson, 2003).

\subsection{Correlación entre el índice de compresión y el índice de plasticidad}

Con los datos de la tabla 4 , se obtuvo el gráfico mostrado en la figura 5 .

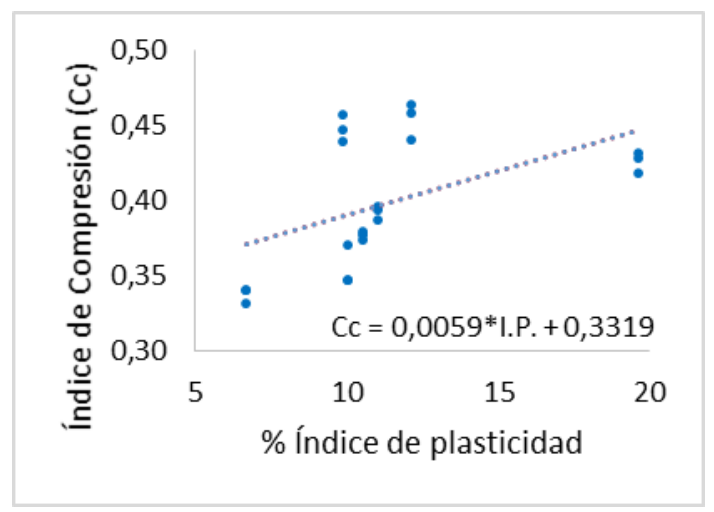

Fig. 5. Correlación entre índice de compresión y el indice de plasticidad. Fuente: Autores.

Del gráfico anterior se obtiene la ecuación de correlación (3):

$$
\mathrm{Cc}=0,0059 *(\text { I.P. }+56,25)
$$

En el análisis realizado entre el índice de compresión y el índice de plasticidad se obtuvo una expresión con un coeficiente de correlación lineal de Pearson $r=0,51$, que permite clasificar el grado de correlación como positiva moderada (Johnson, 2003).

\section{CONCLUSIONES}

El desarrollo de la investigación realizada en los suelos pertenecientes a los perfiles de meteorización derivados de la roca de tipo ígneo perteneciente a la formación geológica Complejo Intrusivo - Extrusivo, presente en el municipio de Ocaña, Norte de Santander, permitió verificar la existencia de una correlación de tipo lineal entre el índice de compresión y las propiedades de plasticidad (límite líquido, límite plástico e índice de plasticidad) de estos suelos.

De las tres ecuaciones de correlación obtenidas, la que brinda un mayor coeficiente de correlación de Pearson es la permite obtener el índice de compresión a partir del límite plástico, expresada en la ecuación 2, por lo que resulta más adecuada al momento de obtener este parámetro de consolidación.

El límite líquido como variable para obtener el índice de compresión, también arrojó un coeficiente de Pearson aceptable, por lo que su uso 
resulta adecuado en la obtención del índice de compresión de acuerdo a la ecuación 1.

El índice de plasticidad fue la propiedad que menor coeficiente de Pearson suministró, por lo que no se recomienda su uso para la obtención del índice de compresión.

Las ecuaciones de correlación encontradas para la determinación del Índice de Compresión a partir de los límites de plasticidad de un suelo representan una ayuda para estimar este parámetro de forma rápida y poder tener una aproximación a los valores de asentamientos por consolidación de los suelos cohesivos de origen residual en la ciudad de Ocaña, no obstante estas correlaciones no remplazan la necesidad de realizar ensayos de consolidación unidimensional que permitan convalidar los resultados de esta investigación.

\section{RECONOCIMIENTO}

Extendemos nuestros agradecimientos a la Universidad Francisco de Paula Santander Ocaña por los recursos facilitados para el desarrollo de esta investigación, en particular al personal del laboratorio de Suelos y Pavimentos.

\section{REFERENCIAS}

Acevedo, E., Flores, O. \& Gómez, E. (2014). "Relación que guarda el índice de compresión de las arcillas del Valle de México con las propiedades índice". XXVII Reunión Nacional de Mecánica de Suelos e Ingeniería Geotécnica.

Ayala C., K. L. (2013). Influencia de las propiedades indice en el indice de compresión en las arcillas del Valle de México, Universidad Nacional Autónoma de México, México. http://www.ptolomeo.unam.mx:8080/jspui /bitstream/132.248.52.100/6176/1/TESIS. pdf (Consultado: 20 de Noviembre de 2018).

Azzouz, A. S. et. al. (1976). Regression Analysis of Compressibility, Soils and Foundation, Volumen 16, No. 2, págs.. 19-29.

Beltran F., S. A. (2015). Incidencia del tipo de ensayo de consolidación en el cálculo de asentamientos en suelos residuales de anfibolita, caso Valle de San Nicolás,
Escuela de Ingeniería de Antioquía, Medellín, Colombia. https://repository.eia.edu.co/bitstream/111 90/2044/1/BeltranSantiago_2015_Inciden ciaTipoEnsayo.pdf (Consultado: $15 \mathrm{de}$ noviembre de 2018)

Crespo, V. (2004). Mecánica de Suelos y Cimentaciones, Quinta edición, Limusa, México.

Cuanalo C., O. A., Gallardo A., R.J., Polanco, G. y Quintero L., L. J. (2016). Problemas geotécnicos en edificaciones desplantadas sobre depósitos aluviales en llanular y penillanuras de inundación. Revista INGENIO, ISSN: 2389-864X Vol. 12.

Das, B. M. (2015). Fundamentos de Ingeniería Geotécnica, Cuarta edición, Cengage Learning, México.

Gallardo A., R. J., Cuanalo C., O. A., Quintero L., L., Muñoz, A. A. y Martínez, C. A. (2017). Análisis del comportamiento de suelos de alta plasticidad con la adición del material de residuo en la fabricación de ladrillo cerámico. REVISTA COLOMBIANA DE TECNOLOGÍAS DE AVANZADA, ISSN: 1692-7257 Vol. 1, No. 29.

Gallardo A., R. J., Gómez P., L. E. y Guillin A., W. F. (2018). Análsisis del comportamiento de suelos de alta plasticidad estabilizados con cementantes. Ecoe ediciones, $1^{\text {a }}$. Ed., Bogotá, Colombia.

García L., R. A., Flórez S., E. N. y Acevedo P., C. (2018). Caracterización térmica de mezclas de arcillas utilizadas en la fabricación de productos de mamposteria para la construcción. REVISTA COLOMBIANA DE TECNOLOGÍAS DE AVANZADA, ISSN: 1692-7257 Vol. 1, No. 31.

Koppula, S. D. (1981). Statistical estimation of compression index, Geotechnical Testing Journal, ASTM, Volumen 4 (2), págs.. 68-73.

Instituto Nacional de Vías (INVIAS). (2012). Especificaciones generales de construcción de carreteras y normas de ensayo para materiales de carreteras. https://www.invias.gov.co/index.php/infor macion-institucional/139-documentotecnicos/1988-especificaciones-generalesde-construccion-de-carreteras-y-normasde-ensayo-para-materiales-de-carreteras (Consultado: 15 de mayo de 2019)

JEG Plaza, MAR Nuñez, (2017) Formación en competencias específicas para la industria 
del software colombiano. Experiencias del uso del aprendizaje basado en proyectos. Revista Colombiana de Tecnologías de Avanzada, ISSN: 1692-7257

Johnson, R. (2003). Estadística Elemental, Tercera edición, Math Learning, México.

Juárez, E. y Rico, A. (2012). Mecánica de suelos, Tomo 1: Fundamentos de la Mecánica de Suelos, Limusa, México.

Nishida, Y. (1956). A brief Note on compression Index of Soils, Journal of Soil Mechanics and Foundations Division, ASCE, Volumen 82, No.SM3, págs.. 1027-1 a 1027-14.

Peck, R. B., Hanson, W. E. y Thornburn, T. H. (2004). Ingeniería de Cimentaciones, Limusa, México.

R García-León, E Florez, C Acevedo (2018). Caracterización térmica de mezclas de arcillas utilizadas en la fabricación de productos de mampostería para la construcción. Revista Colombiana de Tecnologías de Avanzada, ISSN: 1692-7257.

Sáez, E. (2010). Fundamentos de Geotecnia, Pontificia Universidad Católica, Chile.

https://www.u-

cursos.cl/usuario/7c1c0bd54f14c0722cefc

0fa25ea186d/mi_blog/r/FUNDAMENTO

S_DE_GEOTECNIA_SAEZ_(1).pdf

(Consultado: 05 de febrero de 2019)

Servicio Geológico Colombiano (SGC). (2007). Atlas Geológico de Colombia. Servicio Geológico, Primera edición, Bogotá, Colombia.

Sowers, G. B. y Sowers, G. F. (1972). Introducción a la Mecánica de suelos y cimentaciones, Limusa, México.

Terzaghi, K. y Peck, R. B. (1967). Mecánica de suelos en la Ingeniería Práctica, El Ateneo, Segunda edición, Barcelona, España.

L Tangarife, M Sánchez, M Rojas (2017). Modelo de interventoría de tecnologías de información en el área de conocimiento de la gestión del alcance de PMBOK® y alineado con ISO 21500 y COBIT®. Revista Colombiana de Tecnologías de Avanzada, ISSN: 1692-7257.

Molina V., G. M., Hernández L., E. y Castillo R., C. C. (2012). "Determinación de la correlación entre el coeficiente de compresión y propiedades índice en suelos de expansión urbana de Pereira". AVANCES Investigación en Ingeniería, Vol. 9, No. 2.

Xiao, M. (2015). Geotechnical Engineering Design, Wiley Blackwell, edition first, United Kingdom. 